\title{
ARTERIAL INJURIES COMPLICATING ORTHOPAEDIC SURGERY
}

\author{
R. E. Horton, Bristol, England
}

The possibility of accidental injury to an artery in the course of surgical treatment has been known for centuries. Galen described an incident in which a young surgeon damaged the brachial artery in the course of phlebotomy, and commented that most such cases resulted in aneurysm. William Hunter also described arterial injury as a complication of phlebotomy.

In recent years there have been a number of articles in which a variety of arterial injuries following orthopaedic surgery have been described. Many orthopaedic operations are done near major arteries, and often under a tourniquet when an injury can easily be overlooked. It is, perhaps, surprising that with so many operations annually performed, the number of accidents is so low.

\section{INJURY TO MAJOR VESSELS DURING OPERATIONS OF THE LUMBAR SPINE}

Operations on the lumbar spine probably account for most great vessel injuries, and since the first description by Linton and White (1945) a number of publications have appeared. Boyd and Farha (1965) reviewed the literature up to 1963, and were able to find records of twenty-five cases, including two by the author (Horton 1961). However, it seems certain that many cases remain unpublished. When De Saussure (1959) sent a questionnaire to some 3,000 orthopaedic surgeons and neurosurgeons he collected 106 great vessel injuries complicating lumbar disc surgery, with a mortality of 47 per cent. Fifteen were arteriovenous fistulae.

The first patient described by me in 1961 went into early heart failure and was explored on the seventh day. A fistula was found between the right common iliac artery and the right common iliac vein. It was repaired by suture of the vein, but the defect in the artery required a 2-centimetre homograft. This patient was well three years after the operation but has since been lost to follow-up.

The second patient had had a disc operation seven years earlier, and was found to be in heart failure at the time of cholecystectomy. Operation disclosed a fistula between the left common iliac artery and the left common iliac vein. The fistula was divided and the two vessels repaired. Sixteen years after the original disc operation and nine years following repair of the fistula he is free from any disability.

There is no doubt that the great vessels are easily damaged, and it has been suggested that a defect in the anterior longitudinal ligament might explain the ease of its penetration. Bleeding into the disc space occurred in less than half of the cases, and the complication must be suspected if the patient shows an unexplained collapse.

\section{ANEURYSM OF LUMBAR ARTERY FOLLOWING SPINAL FUSION OPERATION}

\section{CASE REPORT}

Case 1-A schoolmistress complained of chronic back pain. She was investigated by myelography in 1962 and treated in a plaster jacket. Later the lower lumbar discs were explored but no abnormality was found. Pain continued and she was treated in a further plaster and, in December 1963, by posterior spinal fusion. At this operation grafts were taken from the iliac crest and applied to the spine from the fourth lumbar vertebra to the second sacral; Wilson plates were bolted on to the spinous processes of the fourth and fifth lumbar and second sacral vertebrae. Immediately she regained consciousness she complained of severe pain in the left leg which continued despite all attempts to relieve it. About a year 
later the left leg showed severe and disabling hyperaesthesia with much muscle wasting. An aortograph (Fig. 1) showed an aneurysm of the left fourth lumbar artery, which was thought to be stretching the roots of the lumbar plexus. The aneurysm was exposed through a transperitoneal approach. A large pulsating swelling was bulging within the psoas muscle. The nerve roots of the lumbar plexus were stretched round the aneurysm. After control of the aorta the aneurysm was incised to release much blood and thrombus. The lumbar artery

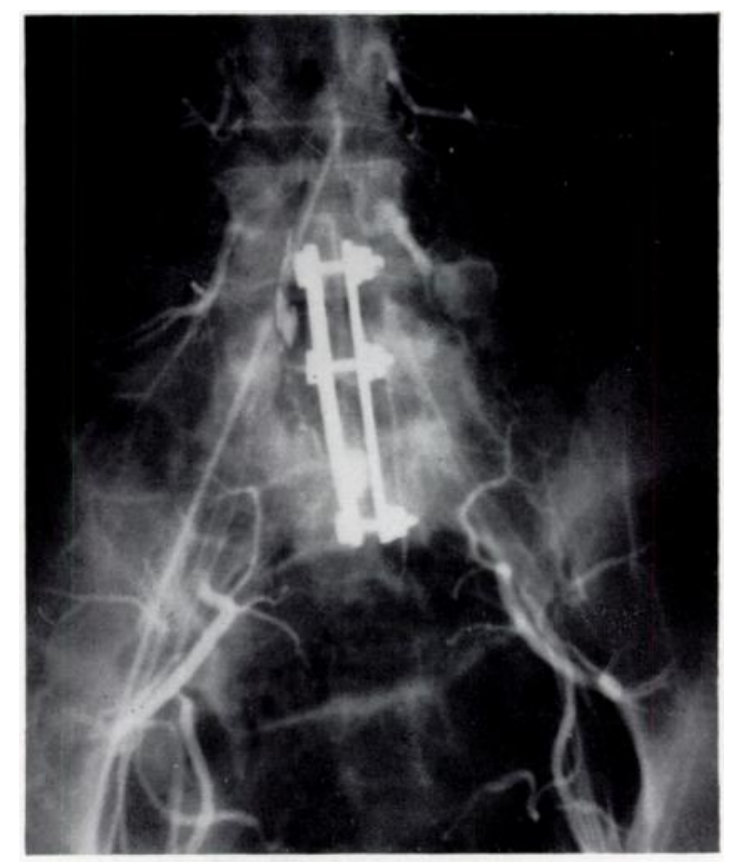

FiG. 1

Case 1-Aortograph. The delayed film shows dilatation of fourth left lumbar artery with an aneurysm about 3 centimetres from its origin.

was ligated at each end. After the operation she continued to have the pain although further aortography showed no recurrence of the aneurysm. There was a possibility of a functional element in this patient though the aneurysm was genuine enough, and further exploration and division of the fourth lumbar root did not help her.

This particular vascular injury does not appear to have been recorded after spinal surgery.

\section{INJURY TO THE ARTERIES IN THE FOOT}

Because most operations on the foot are done under tourniquet an arterial injury can easily be made and not recognised. Webb-Jones (1955) described three aneurysms, of the medial plantar artery after a Steindler operation, of the dorsalis pedis artery after a Lambrinudi operation and of the posterior tibial artery after a triple arthrodesis. All were ligatured and excised. Higgs (1931) described an arteriovenous fistula complicating a triple arthrodesis. Morris and Morse (1966) attempted, but failed, to repair an aneurysm of the posterior tibial artery after a Lambrinudi operation. It is surprising that no successful repair of this artery has been recorded, perhaps because the collateral circulation is thought to make preservation of the posterior tibial artery an unnecessary technical exercise. This is not so (Horton 1968) and although the lack of a posterior tibial artery may not affect an adult, in a growing child it can cause fibrous contracture of the foot and inhibit growth. 
CASE REPORT

Case 2 - A boy was born in 1964 with bilateral talipes equinovarus. In 1966 he had a medial release operation. His mother observed a faint vibrating sensation in the foot after this, but the arteriovenous fistula that had developed was not yet large because it was not noted at elongation of the tendo calcaneus in 1970, also done under tourniquet. Later the same year the fistula was larger because the vibration was more obvious, with an enlarged leg and foot and venous engorgement. An arteriograph showed the fistula involving the posterior tibial artery just proximal to its bifurcation (Fig. 2). At operation the posterior tibial artery was repaired (Fig. 3).

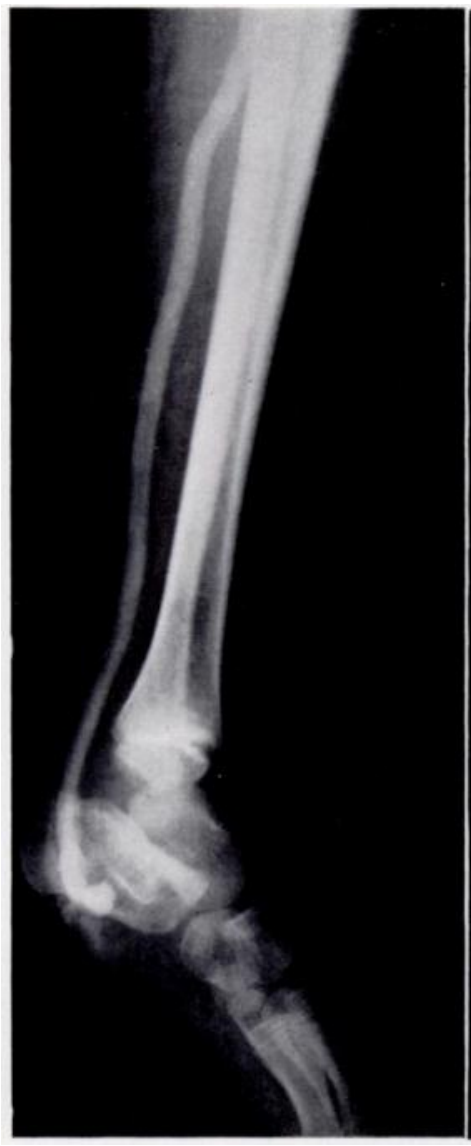

Fig. 2

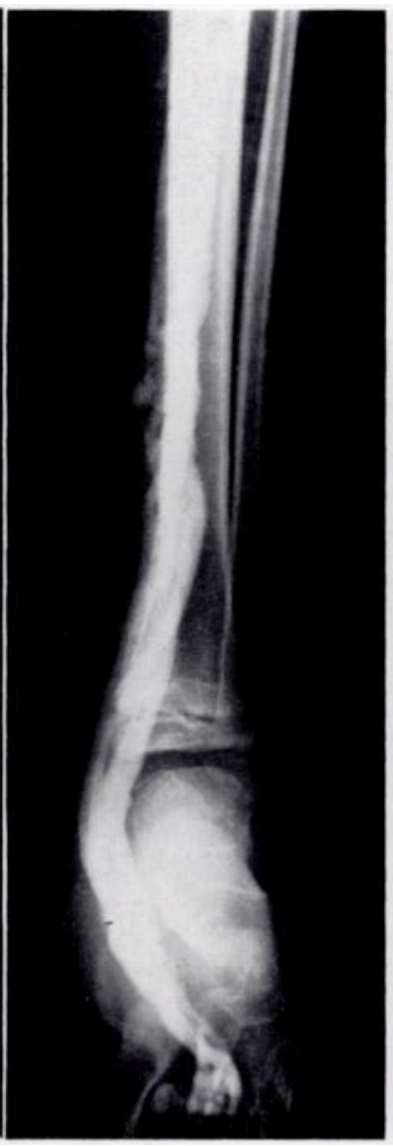

Case 2. Figure 2-Antero-posterior and lateral views of the arteriograph showing the arteriovenous
fistula and the early venous filling. Figure 3-Arteriograph after repair of the fistula.

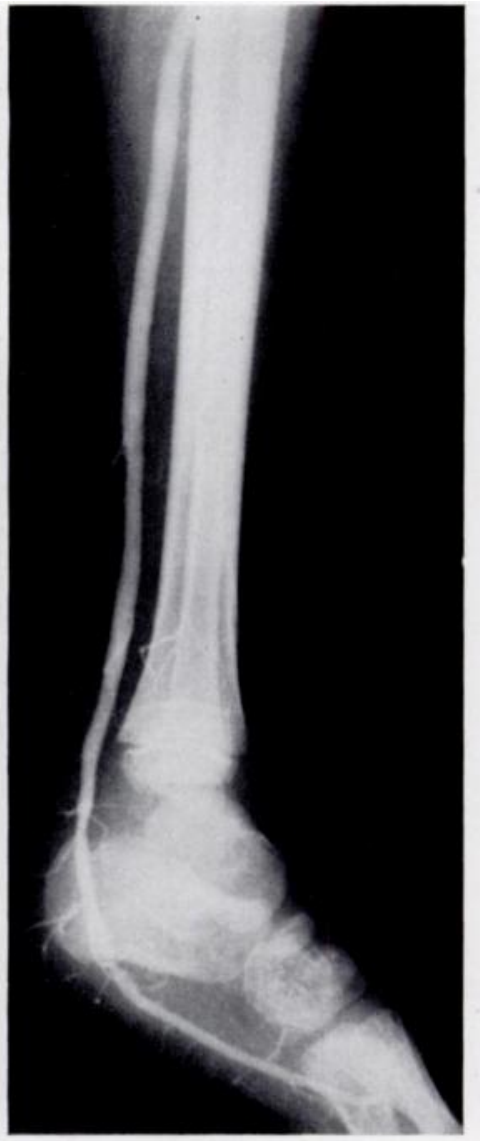

FiG. 3 fistula and the early venous filling. Figure 3-Arteriograph after repair of the fistula.

\section{FAISE ANEURYSM OF THE PROFUNDA FEMORIS ARTERY FOLLOWING NAII. AND PLATE OPERATIONS}

Because it is so near to the femur it is surprising that traumatic aneurysm of the profunda femoris artery after fracture is rare. It has been described by Dameron (1964) after a fracture of the femoral neck treated by a Jewett nail and four screws; by Meyer and Slager (1964), after a subtrochanteric osteotomy fixed with a Blount spline and four screws: and by Bassett and Houck (1964), after osteotomy and plating for slipped epiphysis. In all, the aneurysm was attributed to a projecting screw. Fordyce (1968) described an aneurysm which was explored a month after plating in which the profunda femoris was found to have two holes, perhaps caused by the drill. 


\section{CASE REPORT}

Case 3-A woman aged seventy-two had a McKee pin and plate for a trochanteric fracture. Ten months later she had a sudden severe pain in the thigh and noticed a large pulsatile swelling. Arteriographs (Fig. 4) showed an aneurysm of the profunda femoris artery which was repaired. First the common femoral artery was exposed and controlled, the aneurysm was incised and about half a litre of blood and clot were evacuated. The small hole from the artery to the aneurysm was repaired with a few interrupted fine silk sutures. A projection from the femur at the site of the aneurysm could not be certainly identified as a bone splinter

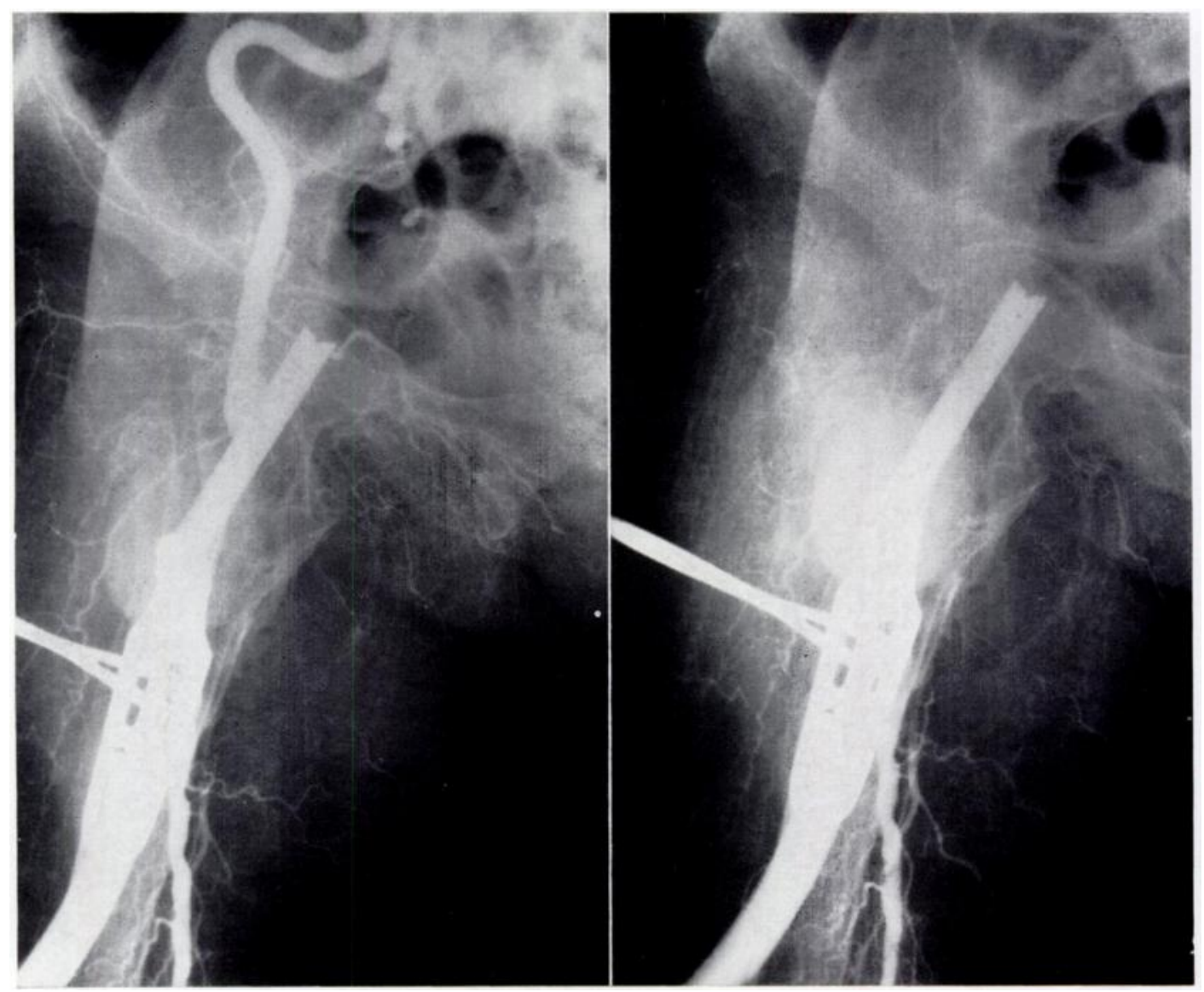

FIG. 4

Case 3-Two radiographs from the arteriograph show the aneurysm of the profunda femoris artery. The proximal part of the femoral artery is obscured by the nail and plate. The superficial femoral artery is atherosclerotic.

or projecting screw, but was probably the latter. The wound healed rapidly. There was no sign of vascular trouble when she was admitted two years later with a fracture of the opposite femur.

A variety of operations have been advised for aneurysm of the profunda femoris artery. It seems clear that it should be repaired if possible, because especially in the older patient atheromatous occlusion of the superficial femoral would be disastrous if the profunda femoris artery had already been ligated. In this case the arteriograph showed the superficial femoral to be severely atherosclerotic (Fig. 4). 


\section{SUMMARY AND CONCLUSIONS}

1. Three new cases of injury to main arteries at orthopaedic operations are described, and a follow-up of two previously described cases is given. The rarity of these accidents is a credit to those engaged in orthopaedic surgery, for many orthopaedic operations are done very close to great vessels, and often under the bloodless conditions of a tourniquet. Nevertheless, it is well to have the risk of great vessel injury in mind because such an accident, although rare, may be a serious threat to life or limb.

2. It is important to repair the damaged artery rather than ligate it because the risk to the growth or viability of the limb is lessened.

3. The aim should be a return to full function with as near normal a vascular anatomy as possible.

\section{REFERENCES}

Bassett, F. H., III, and Houck, W. S., Jun. (1964): False Aneurysm of the Profunda Femoris Artery After Subtrochanteric Osteotomy and Nail-plate Fixation. Journal of Bone and Joint Surgery, 46-A, 583.

Boyd, D. P., and FARHA, G. J. (1965): Arteriovenous Fistula and Isolated Vascular Injuries Secondary to Intervertebral Disk Surgery. Annals of Surgery, 161, 524.

Dameron, T. B., Jun. (1964): False Aneurysm of Femoral Profundus Artery Resulting from Internal-fixation Device (Screw). Journal of Bone and Joint Surgery, 46-A, 577.

DeSaussure, R. L. (1959): Vascular Injury Coincident to Disc Surgery. Journal of Neurosurgery, 16, 222.

Fordyce, A. (1968): False Aneurysm of the Profunda Femoris Artery Following Nail and Plate Fixation of an Intertrochanteric Fracture. Journal of Bone and Joint Surgery, 50-B, 141.

Horton, R. E. (1961): Arteriovenous Fistula Following Operation for Prolapsed Intervertebral Disk. The British Journal of Surgery, 49, 77.

Horton, R. E. (1968): Injury to the Posterior Tibial Artery Complicating Fracture of the Tibia. British Journal of Surgery, 55, 93.

Higgs, S. L. (1931): Arterio-venous Aneurysm of the Posterior Tibial Vessels following Operation for Stabilizing the Foot. Proceedings of the Royal Society of Medicine, 24, 1378.

Linton, R. R., and White, P. D. (1945): Arteriovenous Fistula between the Right Common lliac Artery and the Inferior Vena Cava. Archives of Surgery, 50, 6.

Meyer, T. L., Jun., and Slager. R. F. (1964): False Aneurysm Following Subtrochanteric Osteotomy. Journal of Bone and Joint Surgery, 46-A, 581.

Morris, E., and Morse, T. S. (1966): Aneurysm of the Posterior Tibial Artery After a Foot Stabilization Procedure. Journal of Bone and Joint Surgery, 48-A, 337.

Webb-Jones, A. (1955): Aneurysm After Foot Stabilisation. Journal of Bone and Joint Surgery, 37-B, 440. 